\title{
RADICAL CYSTECTOMY WITH PRESERVATION OF SEXUAL FUNCTION AND URINARY CONTINENCE: DESCRIPTION OF A NEW TECHNIQUE
}

\author{
MIGUEL SROUGI, MARCOS DALL'OGLIO, LUCIANO J. NESRALLAH, HOMERO O. \\ ARRUDA, VALDEMAR ORTIZ \\ Division of Urology, Paulista School of Medicine, Federal University of São Paulo, \\ UNIFESP, São Paulo, SP, Brazil
}

\begin{abstract}
Objective: To describe the original cystoprostatectomy technique which allows the preservation of sexual and urinary function in the majority of treated patients.

Surgical Technique: The described technique presents some details that distinguish it from classic cystectomy: 1) a more efficient control of prostate venous and arterial tributaries; 2) preservation of prostatic capsule and enucleation of prostatic parenchyma, which is removed in block together with the bladder, without violating the vesical neck; 3) no manipulation of the distal urethral sphincteric complex; 4) preservation of seminal vesicles and maintenance of cavernous neurovascular bundles; 5 ) wide anastomosis between the ileal neobladder and the prostatic capsule.

Comments: The proposed maneuvers allow the performance of radical cystectomy with integral preservation of distal urethral sphincter and of cavernous neurovascular bundles, without jeopardizing the oncological principles.
\end{abstract}

Key words: bladder; bladder neoplasms; cystectomy; urinary diversion; urinary reservoirs, continence Int Braz J Urol. 2003; 29: 336-44

\section{INTRODUCTION}

Patients who have an invasive bladder cancer, stages $\mathrm{T}_{2}-\mathrm{T}_{4}$, are currently treated with radical surgery, radiotherapy, chemotherapy or with a combination of these approaches (1). According to available data, radical cystectomy with urinary reconstruction represents the most effective way for treating such cases, accompanied by cure rates that oscillate between $53 \%$ to $80 \%$, when there is no regional or systemic extension of the disease (1).

Despite its therapeutic advantages, radical cystectomy represents a major intervention, accompanied by morbidity rates that should not be disregarded. In addition to the inherent post-operative complications, radical cystectomy presented, in the past,
2 serious drawbacks. Until the final 80s, most of these patients underwent an incontinent cutaneous urinary diversion, which constrained them to bear urine-collecting bags, with all the resultant psychological and social drawbacks. Furthermore, almost all male patients developed erectile dysfunction, which compromised their quality of life.

Upon the introduction of orthotopic intestinal neobladders in the urologic practice (2) and the description of the technique that allowed the preservation of cavernous neurovascular bundles (3), the drawbacks of cutaneous ostomies and sexual dysfunction were both mitigated, but they could not be totally avoided. About $10 \%$ of patients treated in this way maintain severe diurnal incontinence and almost half of cases remain with nocturnal enuresis for ex- 
tended periods (4). On the other hand, even when employing the technique for preserving the cavernous bundles, only $50 \%$ of the treated patients evidence penile erections post-operatively (5).

With the purpose of solving these problems, Spitz et al. described in 1999 an alternative technique of radical cystectomy that preserved sexual, ejaculatory, and urinary functions in treated patients (6). Other studies were subsequently published with the same scope $(7,8,9)$ and all of them contemplated, in a common way, maneuvers intended to maintain the integrity of the distal urethral sphincteric complex, responsible for urinary continence, and the cavernous neurovascular bundles, implied in the sexual function. Despite the significant reduction in risks of urinary incontinence and erectile dysfunction, these techniques presented 2 shortcomings witnessed by us in a small number of treated cases. The block transection of the prostate gland along with the vesical neck is accompanied by a more marked bleeding that the one observed when employing classical techniques of radical cystectomy. For the same reason, preservation of the prostatic parenchyma, common to all such new proposed techniques creates the risk of incomplete removal of vesical neoplasia, when it infiltrates and outgrows the vesical neck. For these reasons, we proposed a new technique of radical cystectomy that aims to preserve the integrity of sexual and urinary functions and that allows a greater control of intraoperative bleeding and a more effective resection of tumors located close to the vesical neck.

\section{SURGICAL TECHNIQUE}

With the patient under general anesthesia, through a wide median abdominal incision, the bladder is separated from the abdomen anterior wall, maintaining, together with the organ, the parietal peritoneum that covers it superiorly. A bilateral pelvic lymphadenectomy is performed, removing the lymph nodes located around the common, external and internal iliac vessels, and close to the obturator vessels. Distal ureters in both sides are dissected and sectioned close to the bladder.

Subsequently, the bladder is laterally released from the pelvic wall and the vesicoprostatic segment is anteriorly dissected up to the prostatic apex. The preprostatic fat is removed, carefully controlling the superficial branch of the deep dorsal vein of penis at the level of the prostatic apex. The intervention proceeds with transection of lateral peritoneal wings, which fix the vesical dome to the pelvic wall. Inside these sheets, we found the vas deferens, and in a more posterior location, the superior vesical arteries, all of which are sectioned and ligated.

Upon releasing the bladder from the structures that involve it anteriorly, superiorly and laterally, the hemostatic control of arterial and venous vessels that involve the prostate begins. Prostatic arteries, located in the vesico-prostatic sulcus on each side, are ligated with 2 large and deep "figure-of- 8 " stitches, with vicryl zero, applied next to the origin of such vessels in the inferior vesical arteries (Figure-1). Next, the 3 venous trunks, one medial and two lateral, which run over the anterior prostate surface, from the deep dorsal vein of penis, are controlled. To accomplish this, 2 parallel and transversal rows of 3 zero vicryl stitches are applied; with the first row located more distally, at about $1.5 \mathrm{~cm}$ from the vesical neck, and other row more proximally, at $0.5 \mathrm{~cm}$ from the neck (Figure-2). These stitches penetrate deeply the prostate capsule and, once they are tied, they control the prostate ascending venous tributaries and the bladder descending branches. They also enable the control of arterial vessels that run in the prostatic capsule. Before incising the prostatic capsule, a third row of 3 zero vicryl stitches is done distally to the previous rows, and are not tied (Figure-2). The anterior portion of the prostatic capsule is incised transversally with an electrocautery, between the first 2 rows of stitches previously tied, until the prostatic parenchyma is reached. Through digital and scissors-aided dissection, the parenchyma is separated from the prostatic capsule, in a maneuver similar to the one performed when an adenoma is enucleated. The urethra is sectioned distally, but the base of the prostate is kept adhered to the vesical neck, forming a single block with the bladder, whose lumen is not violated (Figure-3). Upon the completion of the distal enucleation of the prostate, the more distal capsular stitches are tied and kept repaired. This maneuver allows the definitive control of tributaries of the deep dorsal vein 


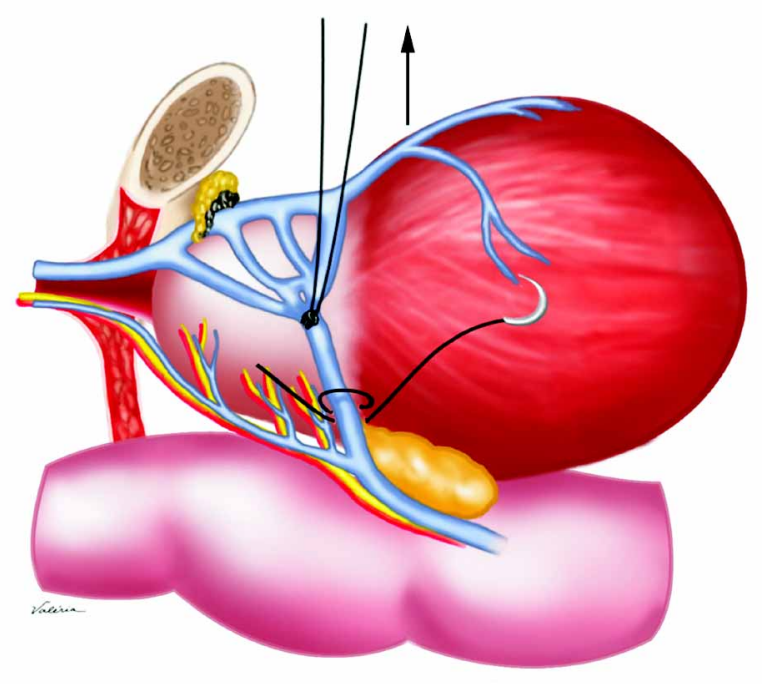

Figure 1 - Double ligation of prostatic artery.

of the penis, which often start to bleed in the capsulotomy's distal margin following the prostatic enucleation. Such intercurrence results from the loosening of the previously tied capsular stitches, due to the enucleation of the adenoma.

At this moment, the anterior manipulation of prostate and bladder is interrupted and the posterior dissection of the block is proceeded. In order to create a correct plane between the bladder and the seminal vesicles, which will be preserved, we repaired the

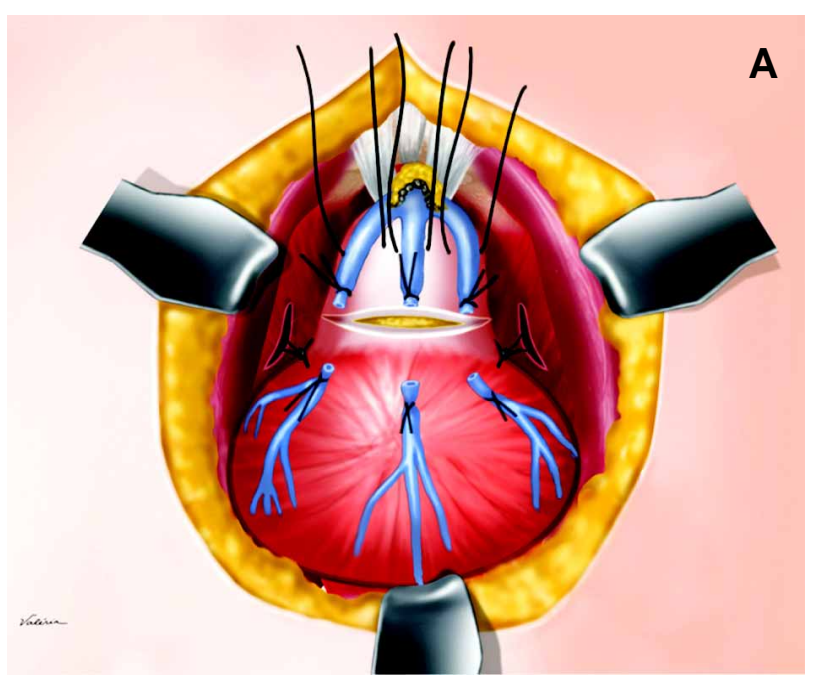

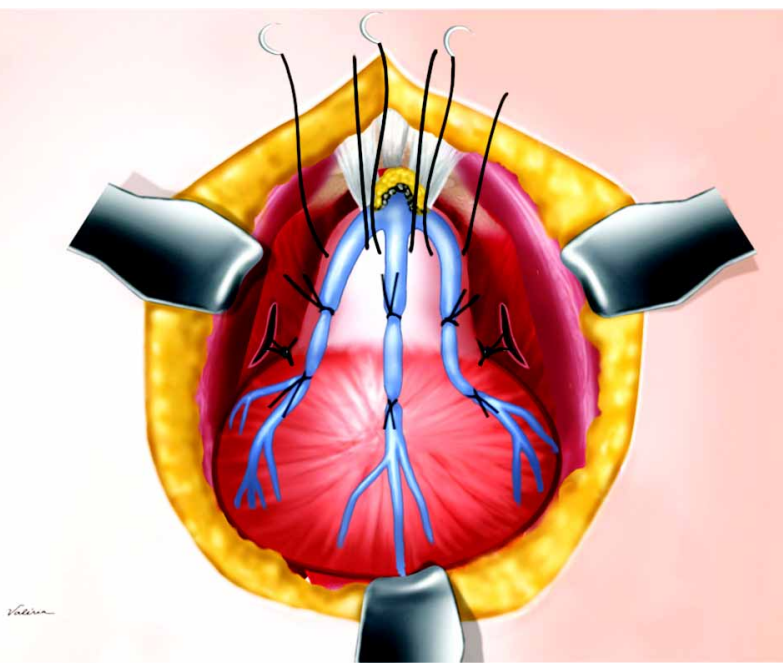

Figure 2 - Control of ascending and descending venous tributaries.

vas deferens in both sides at the posterosuperior surface of the bladder. Through digital and scissors-aided dissection, the surgeon advances in caudal direction between the bladder and the vas deferens, and then anteriorly to the seminal vesicles, until the prostatic base is reached (Figure-4). Resuming the anterior dissection of the specimen and maintaining a small sponge between the bladder and the seminal vesicles, the capsulotomy is completed in its posterior half, with a special precaution to avoid damage to the cav-

Figure 3 - A) Anterior capsulotomy. B) Enucleation of the adenoma.

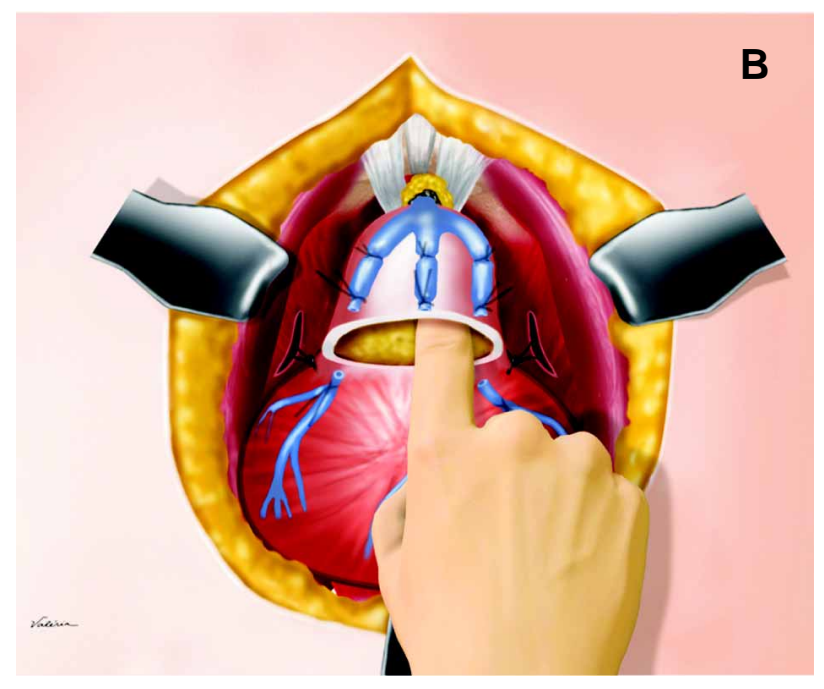




\section{CYSTECTOMY WITH PRESERVATION OF SEXUAL FUNCTION AND CONTINENCE}

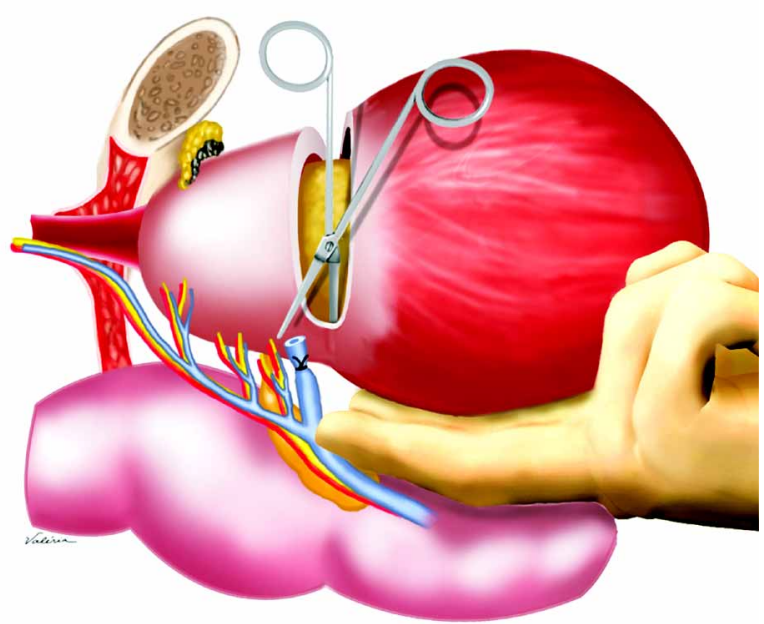

Figure 4 - Detachment of posterior surface of the prostate.

ernous neurovascular bundles (Figure-5). These maneuvers culminate with the complete release of the bladder-prostatic adenoma block, which will be removed, and the distal prostatic capsule, preserved. Samples of tissue from the distal margin of the capsule are removed and submitted to freezing pathologic study in order to confirm the absence of residual neoplasia.

Cystectomy is completed sectioning the 2 lateral vesical pedicles, performed with the aid of Mixter forceps or hemoclamps applied in craniocaudal direction. The specimen formed by the bladder connected to prostatic adenoma is removed, the prostatic cavity and the capsular margins are revised and small bleeding vessels are controlled with electrocautery or with "figure-of-8" 3-zero vicryl stitches.

The intervention proceeds with the construction of an orthotopic ileal neobladder, for which we use Camey II or Studer techniques $(2,10)$. Once the neobladder is done and double-J catheters are inserted in both ureters, the anastomosis between the neobladder and the remaining distal prostatic capsule is performed (Figure-6). This anastomosis is made with a continuous 2-zero vicryl suture, and before its completion a $20 \mathrm{~F}$ Foley urethral catheter is placed in the neobladder, and the distal ends of both double-J catheters are tied to it.

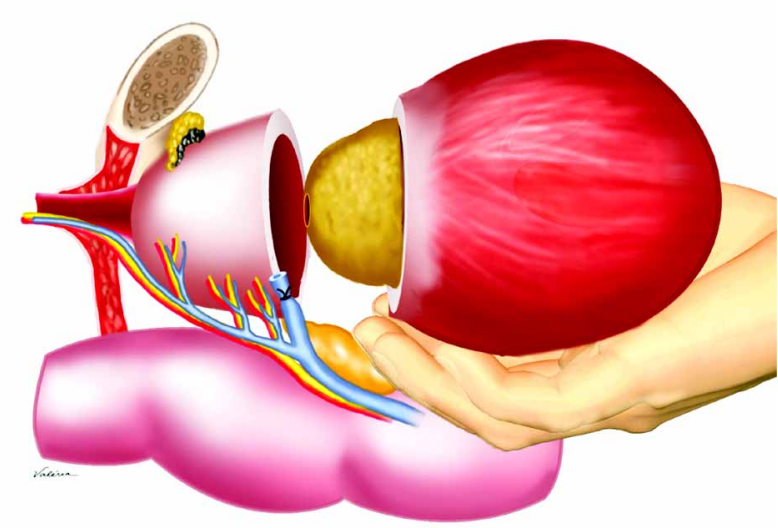

Figure 5 - Release of bladder-adenoma block, following posterior capsulotomy.

Surgery is finished with the installation of continuous suction drains at the level of the anastomosis between the neobladder and the prostate and near the sites of ureteral implantation. These drains are maintained until the $7^{\text {th }}$ post-operative day and the Foley catheter, tied to the double-J catheters, are removed on the $20^{\text {th }}$ day after the intervention.

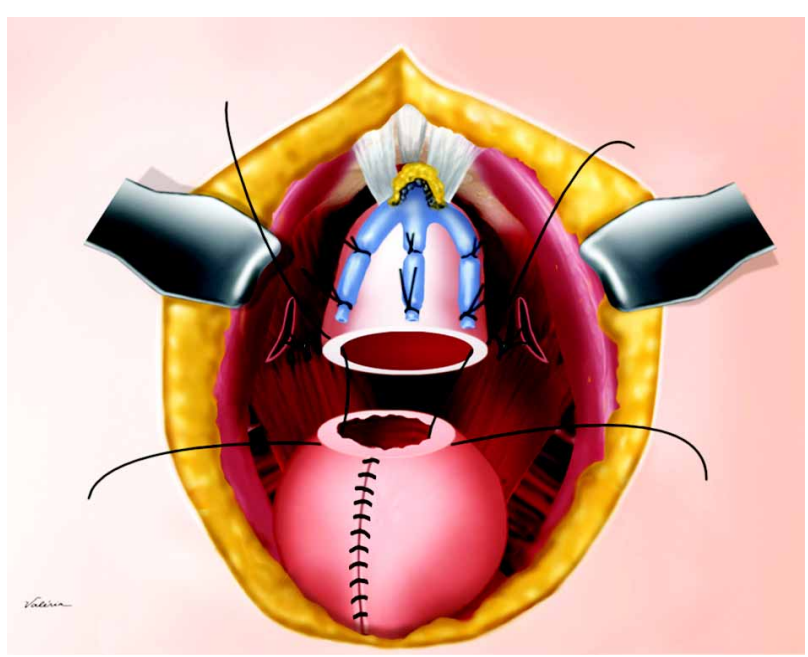

Figure 6 - Anastomosis between the neobladder and the prostatic capsule. 


\section{COMMENTS}

In this work we present an original alternative technique for radical cystectomy, which allows integral preservation of urinary continence and reduces substantially the risks of sexual impotence. In an initial group of 6 treated patients, 5 presented complete diurnal and nocturnal continence immediately after removing the urethral catheter, 3 referred penile erections on the first month and none evidenced positive surgical margins at the level of vesical neck or prostatic parenchyma.

In contrast to the classical radical cystoprostatectomy technique, this method preserves the prostatic capsule, the cavernous neurovascular bundles and is not accompanied by manipulation of the distal urethral sphincteric complex. For such reasons, this technique, which could be referred to as cysto-adenomectomy, has a highly favorable impact over the maintenance of urinary and sexual functions post-operatively. Another advantage of this technique is the fact that it is accompanied by a block removal of bladder and prostatic parenchyma, reducing the risks of incomplete removal of the vesical neoplasia, when it invades the vesical neck and the prostate by intraluminal direct extension.

Under a surgical perspective, this method incorporates maneuvers that allow a quite efficient control of the anterior periprostatic venous trunks and the lateral prostatic arteries (11), significantly reducing intra-operative bleeding. As a matter of fact, none of the 6 patients treated up to now, required blood transfusions during or after surgery.

The only drawback of the cystoadenomectomy technique, compared with the classic radical cystectomy, is that it does not remove a prostate cancer when this tumor is coincidentally present in addition to the vesical neoplasia (12). To minimize this problem, the cysto-adenomectomy technique must be indicated when the existence of a prostate cancer is highly unlikely, that is, in patients with medical examination and normal serum levels of prostatic specific antigen or, in case of doubt, with negative pre-operative prostatic biopsy.

The first proposal about preservation of sexual and ejaculatory function in radical cystectomy was made in 1999 (6). These authors described a technique that removed the anterior half of the prostate and preserved its posterior portion. After that, 3 more studies were published with the same objective, all of them proposing the preservation of the prostate and bladder resection with distal transection of the specimen at the level of the vesical neck $(7,8,9)$. Despite highly elevated rates of maintenance of sexual and urinary function observed with these techniques, they presented, as a common feature, the risk of violating the bladder tumor and producing positive distal margins, when the neoplasia reaches the vesical neck. This risk was reduced by Colombo et al. (9) and by Vallancien et al. (8) who performed the endoscopic resection of the vesical neck and the prostate previously, but even then, the potential risk of violating the neoplasia persists when the vesical neck is sectioned transversely. In our technique, this possibility is minimized due to the block removal of prostatic parenchyma, vesical neck and bladder.

Another advantage of this cystoadenomectomy technique in relation to other published approaches is that it implies in removing the specimen in one stage. Both previous endoscopic resection (9) and that performed at the moment of cystectomy (8) increase the length and morbidity of the intervention.

If the ongoing study by our group confirms better preservation of urinary continence and a lower incidence of post-operative sexual dysfunction, this technique may become a preferential method for performing radical cystectomy in men with invasive bladder cancer. In such cases, the method could be employed every time that the presence of a primary prostate cancer is previously ruled out, and when there is no extensive secondary involvement of the prostate gland from the vesical neoplasia.

\section{REFERENCES}

1. Sternberg CN: Current perspectives in muscle invasive bladder cancer. Eur J Cancer 2002, 38: 460467.

2. Barre PH, Herve JM, Botto H, Camey M: Update on Camey II procedure. World J Urol. 1996, 14: 27 28. 
3. Schlegel PN, Walsh PC: Neuroanatomical approach to radical cystoprostatectomy with preservation of sexual function. J Urol. 1987, 138: 1402-1406.

4. Soulie M, Seguin P, Mouly P, Thoulouzan M, Pontonnier F, Plante P: Assessment of morbidity and functional results in bladder replacement with Hautmann ileal neobladder after radical cystectomy: a clinical experience with highly selected patients. Urology 2001, 58: 707-711.

5. Miyao N, Adachi H, Sato Y, Horita H, Takahashi A, Masumori N, et al.: Recovery of sexual function after nerve-sparing radical prostatectomy or cystectomy. Int J Urol. 2001, 8:158-164.

6. Spitz A, Stein JP, Lieskovasky G, Skinner DG: Orthotopic urinary, diversion with preservation of erectile and ejaculatory function in man requiring radical cystectomy for nonurothelian malignancy: a new technique. J Urol. 1999, 161: 1761-1764.

7. Horenblas S, Meinhardt W, Ijzerman W, Moonen LFM: Sexuality preserving cystectomy and neobladder: initial results. J Urol. 2001, 166: 837-840.

Correspondence address:

Dr. Miguel Srougi

Rua Peixoto Gomide 2055/81

01409-003, São Paulo, SP, Brazil

Fax: + $55113257-9006$

E-mail: srougi@attglobal.net

\section{EDITORIAL COMMENT}

Srougi et al. adapted a few technical maneuvers acquired from years of performing radical and simple prostatectomies and applied them to a cystoprostatectomy with orthotopic neobladder. The objective is to preserve sexual function and improve urinary continence. In my experience patients have excellent daytime continence $(<5 \%$ wear pads) although $15 \%$ empty by intermittent catheterization. Nighttime incontinence is infrequent since most of my patients wake up at least once per night. I have elected to taper the ileum at the site of the urethral
8. Vallancien G, El Fettouh HA, Cathelineau X, Baumert H, Fromont G, Guillonneau B: Cystectomy with prostate sparing for bladder cancer in 100 patients: 10 -year experience. J Urol. 2002, 168: 2413-2417.

9. Colombo R, Bertim R, Salonia A, Da Pozzo LF, Montorsi F, Brausi M, et al.: Nerve and seminal sparing radical cystectomy with orthotopic urinary diversion for selected patients with superficial bladder cancer: an innovative surgical approach. J Urol. 2001, 165: 51-55.

10. Modersbacher S, Hechreiter W, Burkhard F, Thalmann GN, Danuser H, Markwalder R, et al.: Radical cystectomy for bladder cancer today - a homogenous series without neoadjuvant therapy. J Clin Oncol. 2003, 21: 690-696.

11. Srougi M, Dall'Oglio MF, Bomfim AC, Andreoni C, Cury J, Ortiz V: An improved technique for bleeding control during simple retropubic prostatectomy. BJU Int (in press).

12. Chun TY: Coincidence of bladder and prostate cancer. J Urol. 1977, 157: 65-67.

Received: April, 2003

Accepted: May, 2003 anastomosis which may add to the functional urethral length. I am not certain how important this is.

The issue of preserving erectile function is an important one for a relatively small subset of men who have a cystoprostatectomy and neobladder. The percentage of men who are candidates for this prostate capsule sparing is relatively low among all of the men I evaluate for surgery. The majority of men is older or has advanced local disease and thus they are impotent or the extent of disease makes invasion of the prostate a concern. I believe the patient must be 
one who understands the need for subsequent careful monitoring - not only for urothelial cancer but for adenocarcinoma of the prostate.

The men who are most likely to benefit from these modifications of the standard cystoprostatectomy are younger men who have recurrent or persistent high grade Ta, CIS, or T1 bladder cancer and have failed intravesical therapy. Once tumor at the bladder neck (?) and prostatic urethra is excluded they might be reasonable candidates for this approach.

There are some trade-offs when comparing the standard procedure in which the entire prostate and seminal vesicles are removed with Srougi's modification which leaves the prostate capsule and seminal vesicles. This is not much different however, from leaving the neurovascular bundles, bladder neck, and the distal seminal vesicles during a radical prostatectomy with the desire to improve the chance of retaining normal pre-op erectile function. Each case must be carefully judged based on pre and intraoperative findings as well as a variety of patient issues such as age and erectile function before surgery.

\author{
Mark S. Soloway \\ Chairman, Department of Urology \\ University of Miami School of Medicine \\ Miami, Florida, USA
}

\section{EDITORIAL COMMENT}

En bloc removal of the bladder, prostate, ampullae of the vasa deferentia and seminal vesicles is now the paradigm treatment for muscle invasive and recurrent high grade urothelial carcinomas. However, largely due to significant associated morbidities and only modest cure rates when applied as a monotherapy, initial acceptance of this procedure was not broad. Over the past 30-years both medical and urologic oncologists have made dramatic strides to improve the adverse consequences of effectively treating urothelial cancer. Medical oncologists have graduated patients from non-effective, single-agent chemotherapy to the latest less-toxic but efficacious combination of paclitaxel, carboplatin and gemcitabine. With improved survival, urologic oncologists have modified their surgical execution to reduce morbidity and improve the social, sexual and psychological implications of radical cystectomy. Lower urinary tract reconstruction has evolved from simple cutaneous ureterostomies and ileal conduits to continent cutaneous urinary reservoirs, and most recently the continent orthotopic neobladders. Today men and women can safely undergo orthotopic lower urinary tract reconstruction to the intact native urethra while preserving the erectile nerve bundles and importantly, the pelvic plexus supplying these nerve bundles. This has given witness to a dramatic improvements in both the longevity and quality of our patients' lives.

For continuing these forward strides in surgical techniques with their described method, a modification of that previously described by the USC group (authors' reference 6), the authors are to be commended. And we are sure that many innovative surgeons will continue to refine this technique to provide even better outcomes for the patient of tomorrow.

But in this search for minimal morbidity, let's not forget in whom and for what reason the vast majority of radical cystectomies are performed. Today the average male patient requiring cystectomy for bladder cancer is in his sixth decade of life when the reproductive necessity of preserving ejaculatory function has generally long since past. In the end, this 
is the one morbidity of standard nerve sparing radical cystoprostatectomy that we see as preserved through this technique, and this is accomplished with a questionable overall improvement in life's quality for the vast majority of men with urothelial carcinoma. We are further puzzled as to the mechanism of antegrade ejaculation following the removal of a functional bladder neck and the necessity for its coordinated closure to provide antegrade emission of deposited seminal fluids.

With a very conscious recognition of the anatomic location of the pelvic plexus lateral to the seminal vesicles and its nervous supply to the erectile nerve bundle as depicted in authors' Figure-1, maintaining the erectile nerve bundles should be no more difficult during radical cystoprostatectomy than radical prostatectomy. We commonly perform retrograde release of the nerve bundles from apex to base during radical cystoprostatectomy as we perform it during radical prostatectomy. Similar to the reports of others, this approach has allowed us to preserve erectile function at rates similar to that seen following isolated radical prostatectomy (1). While this approach obviates ejaculatory function, is this truly an issue for the majority of patients undergoing radical cystoprostatectomy?

We then re-focus on the patients in whom the majority of these surgeries are performed; sixty-yearold men, who also have the highest incidence of prostate cancer (2). Furthermore, we now recognize that a significant number of prostate cancers exist in men with serum PSA below $3 \mathrm{ng} / \mathrm{ml}$, the majority of which are clinically significant $(3,4)$. The benefits of a radical cystectomy which preserves the posterior lateral zones of the prostate might quickly fade for the patient and physician alike when the serum PSA starts rising and there is limited hope of performing completion prostatectomy and reconstruction of the orthotopic neobladder.

The authors' approach is certainly appealing when ejaculatory preservation is a quality of life issue. We however caution that this select patient is few and far between. For the young (20 to 30 year-old) male with benign bladder disease necessitating cystectomy (refractory cystitis glandularis) or nonurothelial carcinoma away from the bladder neck in whom fertility is an issue, this technique, offering ejaculatory preservation, even if ejaculation is retrograde into the neobladder where it can be harvested, is alluring. For all others we feel there is little benefit to be gained by this technique over a properly performed nerve sparing cystoprostatectomy

\section{References}

1. Ghavamian R, Zincke H: An updated, simplified approach to nerve-sparing radical retropubic prostatectomy. BJU Int. 1999; 84:160-163.

2. Sarma AV, Schottenfeld D: Prostate cancer incidence, mortality, and survival trends in the United States: 1981-2001. Semin Urol Oncol. 2002; 20:3-9

3. Recker F, Kwiatkowski MK, Huber A, Stamm B, Lehmann K, Tscholl R: Prospective detection of clinically relevant prostate cancer in the prostate specific antigen range 1 to $3 \mathrm{ng} . / \mathrm{ml}$. combined with free-to-total ratio $20 \%$ or less: the Aarau experience. J Urol. 2001; 166: 851-5

4. Ward JF, Bartsch T, Sebo TJ, Pinggera G-M, Blute ML, Zincke H: Pathologic characterization of prostate cancers with a very low serum prostate specific antigen $(0-2 \mathrm{ng} / \mathrm{mL})$ incidental to cystoprostatectomy: Is PSA a useful indicator of clinical significance? Urol Oncol (in press).

Dr. John F. Ward Fellow in Uro-Oncologic Surgery Department of Urology, Mayo Medical School Rochester, Minnesota, USA

Dr. Horst Zincke Professor of Urology, Mayo Medical School Consultant, Department of Urology Rochester, Minnesota, USA 


\section{EDITORIAL COMMENT}

The concept of preservation of the prostate at the time of cystectomy for bladder cancer is not new and has been applied sporadically since early in the twentieth century. I first preserved the prostate capsule in 1985 using a technique somewhat similar to Srougi and subsequently in several carefully selected patients. I have been reluctant to publish these cases due to the uncertainty as to whether this technique will be viable in the long term. Clearly, interest in this technique has escalated with this recent report and the larger series of Vallancien et al. from France (1).

While there are some early signs that this technique may be beneficial for some patients, the benefits versus the risks of the technique must be addressed before its widespread use. The benefits include probable decrease in blood loss, probable improved continence, and probable improved erectile function recovery. I emphasize the "probable" aspect because the degree of improvement is certainly unquantified, despite the initial observations of Srougi and my experience as well. A validated Quality of Life instrument suitable to quantify erectile function and incontinence after a cystectomy and neobladder surgery is not currently available; thus the magnitude of improvement from this technique will be debatable until studied in an appropriate fashion.

The risks are also unquantified. Using wholemount step-sectioning of the prostate, it has been determined that approximately $40 \%$ of bladder cancer cystectomy patients may harbor unsuspected urothelial carcinoma in situ in the prostatic urethra or prostatic ducts $(2,3)$. Similarly, $40 \%$ to $50 \%$ of patients have unsuspected adenocarcinoma of the prostate (most of which are small and of uncertain clinical significance) (3). Since the patient populations with urothelial and adenocarcinomas do not necessarily overlap, $40 \%$ to $80 \%$ of patients might have a neoplasm in the prostate which may make it unwise to leave the prostate capsule behind. Much of this risk may be obviated by careful patient selection and presurgical screening for prostate cancers as suggested by Srougi, and a frozen section of the adenomatous tissue removed from the prostate. If the frozen section reveals either urothelial or adenocarcinoma at the time of surgery, the prostate capsule and seminal vesicle could be removed. If the frozen section is benign, the neobladder could be sewn to the prostate capsule as described in Srougi's report.

As with many procedures in oncology, the evolution is towards smaller, less extensive operations that still reliably eliminate the cancer but preserve better function (e.g., lumpectomy and radiation for breast cancer or nerve-sparing radical prostatectomy). Maybe it is time for a more critical study of a tailored radical cystectomy for urothelial cancer. Careful patient selection will unquestionably be the most important aspect.

\section{References}

1. Vallancien G, Abou El Fettough H, Cathelineau X, Baumert H, Fromont G, Guillonneau B: Cystectomy with prostate sparing for bladder cancer in 100 patients: 10-year experience. J Urol. 2002; 168: 2413-7.

2. Wood DP, Montie JE, Pontes JE, VanderBrug Medendorp S, Levin HS: Transitional cell carcinoma of the prostate in cystoprostatectomy specimens removed for bladder cancer. J Urol. 1989; 141: 346-9.

3. Mahadevia PS, Koss LG, Tar IJ: Prostatic involvement in bladder cancer: Prostate mapping in 20 cystoprostatectomy speciems. Cancer 1986; 58: 2096102.

\author{
Dr. James E. Montie \\ Chairman, Dept Urology, Univ of Michigan \\ Valassis Professor of Urologic Oncology \\ Ann Arbor, Michigan, USA
}

